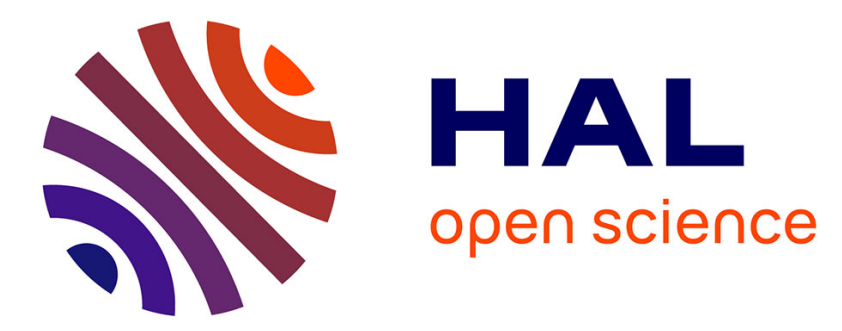

\title{
Are sleep paralysis and false awakenings different from REM sleep and from lucid REM sleep? A spectral EEG analysis
}

Greta Mainieri, Jean-Baptiste Maranci, Pierre Champetier, Smaranda LeuSemenescu, Ana Gales, Pauline Dodet, Isabelle Arnulf

\section{To cite this version:}

Greta Mainieri, Jean-Baptiste Maranci, Pierre Champetier, Smaranda Leu- Semenescu, Ana Gales, et al.. Are sleep paralysis and false awakenings different from REM sleep and from lucid REM sleep? A spectral EEG analysis. Journal of Clinical Sleep Medicine, 2021, 17 (4), pp.719-727. 10.5664/jcsm.9056 . hal-03390728

\section{HAL Id: hal-03390728 \\ https://hal.sorbonne-universite.fr/hal-03390728}

Submitted on 21 Oct 2021

HAL is a multi-disciplinary open access archive for the deposit and dissemination of scientific research documents, whether they are published or not. The documents may come from teaching and research institutions in France or abroad, or from public or private research centers.
L'archive ouverte pluridisciplinaire HAL, est destinée au dépôt et à la diffusion de documents scientifiques de niveau recherche, publiés ou non, émanant des établissements d'enseignement et de recherche français ou étrangers, des laboratoires publics ou privés. 
Are Sleep Paralysis and False Awakenings Different from REM Sleep and from Lucid REM Sleep? A Spectral EEG Analysis

Greta Mainieri, MD1,2; Jean-Baptiste Maranci, MD1,3,4; Pierre Champetier, MS1,4,5; Smaranda LeuSemenescu, MD1; Ana Gales, MD; Pauline Dodet, MD1; Isabelle Arnulf MD, PhD1,3,4

${ }^{1}$ Sleep Disorder Unit, Pitié-Salpêtrière University Hospital, AP-HP, Paris, France

2Department of Medical and Surgical Sciences and Advanced Technologies "G.F. Ingrassia", University of Catania, Catania Via S. Sofia 78, Italy

${ }^{3}$ Sorbonne University, Paris, France

${ }_{4}^{4}$ Paris Brain Institute, Paris, France

${ }^{5}$ Ecole Normale Supérieure Paris-Saclay, Université Paris-Saclay, France

Keywords: sleep paralysis, false awakenings, REM sleep

Submitted to: JCSM, version \#1

Correspondence to: Isabelle Arnulf, Service des Pathologies du Sommeil, Hôpital Pitié-

Salpêtrière, 47-83 Boulevard de l'Hôpital, 75013 Paris, France ; Email: isabelle.arnulf@aphp.fr;

Phone: 33142167702 ; Fax: 33142167700

\section{Statements}

- All authors have seen and approved the manuscript

- None of the authors has any conflict of interest relative to this study

- Number of tables: 2; Number of figures: 3

- Abstract count words: 248 words; Manuscript count words: 4222 words 
Abbreviation list:

EEG: electroencephalography

EMG: electromyography

EOG: electro-oculography

MSLT: multiple sleep latency tests

SOREMP: sleep onset in REM period

REM: rapid eye movement

REMs: rapid eye movements 


\section{Abstract}

Study objectives: To determine the polysomnography characteristics during sleep paralysis, false awakenings and lucid dreaming (which are states intermediate to REM sleep and wake but exceptionally observed in sleep laboratory).

Methods: In 5 subjects, we captured 5 episodes of sleep paralysis (2 time-marked with the ocular left-right-left-right code normally used to signal lucid dreaming, 1 time-marked by an external noise and 2 retrospectively reported) and 2 episodes of false awakening. The sleep coding (using three seconds mini-epochs) and spectral EEG analysis were compared during these episodes and normal REM sleep as well as wakefulness in the same four among these five subjects, and vs. lucid REM sleep in four other patients with narcolepsy.

Results: During episodes of sleep paralysis, $70.8 \%$ of mini-epochs contained theta EEG rhythm, (vs. $89.7 \%$ in REM sleep and $21.2 \%$ in wakefulness), $93.8 \%$ contained chin muscle atonia (vs. $89.7 \%$ in REM sleep and $33.3 \%$ in wakefulness) and $6.9 \%$ contained rapid eye movements (vs. $11.9 \%$ in REM sleep and $8.1 \%$ in wakefulness). The EEG spectrum during sleep paralysis was intermediate between wakefulness and REM sleep in the alpha, theta and delta frequencies, whereas the beta frequencies were not different between sleep paralysis and normal REM sleep. The power spectrum during false awakening followed the same profile as in sleep paralysis.

Conclusions: The predominant theta EEG rhythm during sleep paralysis and false awakenings (with rare and lower alpha rhythm) suggests that the brain during sleep paralysis is not in an awake but in a dreaming state. 


\section{BRIEF SUMMARY}

Current Knowledge/Study Rationale: Sleep paralysis is commonly viewed as an awake brain with a complete muscle atonia, but EEG analysis during sleep paralysis is exceptional.

Study Impact: Our polysomnography and spectral analysis of time-marked sleep paralyses suggest that the brain during sleep paralysis is not in an awake but in a dreaming state. This may explain why hallucinations are often associated with sleep paralysis. 


\section{INTRODUCTION}

Sleep paralysis is classified within the REM sleep parasomnias, as a transient sleep/wake phenomenon occurring when entering into or awakening from REM sleep, with elements of REM sleep persisting into wakefulness. ${ }^{1,2}$ In particular, the REM sleep-associated muscle atonia seems to persist in an awake brain, leading to the often distressing experience of being incapable to move. ${ }^{3}$ Several historical descriptions and iconic representations delineate a picture of a conscious brain in a still 'sleeping' body. The EEG, EOG and EMG features (awake, asleep, or admixture of both stages) during sleep paralysis have been challenging to document, as it is difficult to capture this brief and unpredictable experience in a sleep laboratory. However, some rare sleep paralyses have been documented during polysomnography in healthy subjects ${ }^{4,5}$ and in patients with narcolepsy. ${ }^{6,7}$ In healthy subjects, only episodes elicited by forced awakenings have been studied, using a sleep wake schedule known to facilitate sleep onset REM periods [SOREMPs]. In these instances, elements of REM sleep and wakefulness intermingled in two main patterns: one scored as REM sleep despite abundant trains of alpha rhythms, sometimes interrupted by rapid eye movements (REMs); the other scored as wakefulness with appearance of atonia plus muscle twitches. ${ }^{4,5}$ In patients with narcolepsy, the polysomnography showed features intermediate between REM sleep and wakefulness. ${ }^{7,8}$ In a single case to date, spectral EEG analysis definitely settled the episode as an intermediate state between wake and REM sleep. ${ }^{6}$

Sleep paralysis may sometimes be linked to false awakening, ${ }^{1}$ a state characterized by the perception of waking up in a familiar place and starting a normal daytime routine, only to later discover of having been trapped in one's own dream or to realize of having dreamt. ${ }^{9,} 10$ This state may also be referred to as a "dreamed" awakening. Only one report describes the polysomnography features of a SOREMP hallucination without 
sleep paralysis, ${ }^{11}$ later referred to as a false awakening by other authors. ${ }^{9}$ We incidentally had the opportunity to monitor during polysomnography five episodes of sleep paralysis, one of which continued into a false awakening, as well as an episode of isolated false awakening, and to better characterize the associated EEG pattern.

\section{METHODS}

\section{Recordings and polysomnography analysis}

EEG monitoring included Fp1-A2, C3-A2, O1-A2 cortical channels, left and right electro-oculogram (EOG), chin and tibialis anterior muscle surface electromyography (EMG) for all participants, electrocardiography, pulse oximetry for almost all patients, nasal pressure, recording of tracheal sounds through a microphone placed at the surface of the trachea, thoracic and abdominal belts, as well as EEG-synchronized infrared video-monitoring and ambiance microphone recordings for Patient \#3. In two patients experiencing sleep paralysis (Patient \#1 and Patient \#3), the exact beginning of the episode could be accurately estimated. For them, we divided the 30-second epochs into 10 mini-epochs of 3 seconds each, analyzing each mini fragment for the presence of alpha/theta background activity on EEG, evaluated on O1-A2 cortical channel (we considered the mini-epoch as having alpha or theta when a rhythm was present for $>50 \%$ of the mini-epoch), muscle atonia or increased muscle tone on chin and legs EMG, as well as REMs presence or absence on the EOG. In Patient \#1 who experienced the sleep paralysis during the multiple sleep latency test (MSLT), the cumulative fragments of the two episodes were of comparable duration with the periods of normal REM sleep and quiet wakefulness in the MSLT. Therefore, we analyzed the totality of normal REM sleep epochs outside the sleep paralysis experiences and all fragments of quiet wakefulness in the MSLT. In Patient \#3, the episode of sleep paralysis occurred during the first REM sleep episode of the night, so that we had to match some normal 
REM sleep and eyes-closed wakefulness period of comparable duration. Analyzing epochs of normal REM sleep and wakefulness all over the night would have resulted in a much greater amount of mini-epochs in comparison with the paralysis episode. For this reason, we assigned a crescent numeration to all mini-epochs of REM sleep and wakefulness throughout the night and randomly draw a corresponding number of miniepochs, exactly matching the duration of the sleep paralysis episode. Arousals during REM sleep, including prominent fast activity and tonic increase in chin EMG, have been excluded from the analysis. We then compared the polysomnography elements associated with sleep paralysis to normal REM sleep and quiet wakefulness of the same subjects. Plus, we compared the elements obtained during sleep paralysis with those obtained during lucid REM sleep in 4 narcolepsy patients with lucid dreaming, chosen as "control" group and previously described in other studies from our centre. ${ }^{12,13}$ This particular group of patients carries the advantage of providing a clear time-frame of the episodes (thanks to the ocular coding), giving the opportunity to compare sleep paralysis and false awakenings to another peculiar state of "consciousness while dreaming", unique in its kind, such as lucid dreaming. The same 3 -seconds analysis was applied to the fragments of lucid REM sleep. A $\chi^{2}$ test was performed to compare the categorical variables (presence of alpha/theta EEG, EMG activity, presence of REMs at the EOG) in the studied sleep states. Statistical significance was set at $\mathrm{p}$ value $<0.05$. Statistical analyses were performed with STATA ${ }^{\circledR}$ version 16.0 .

\section{Spectral EEG analysis}

We extracted non-overlapping samplings of EEG recordings (sample frequency of 256 $\mathrm{Hz}$ ) of sleep paralysis periods for 3 patients and false awakenings for 2 patients, matching them with fragments of quiet closed-eyes wakefulness and of normal REM sleep of corresponding duration. We excluded ocular artifacts by means of visual 
inspection in all samples. The samples were exported to perform spectral analysis on each electrode channel with Fast Fourier Transform. For each EEG activity, the power spectrum was estimated using the Welch averaged periodogram method using the Matlab Toolbox EEGLAB. For each state (sleep paralysis, false awakening, normal wakefulness and normal REM sleep), we cumulated all the fragments of the patients and performed the mean of the three EEG channels. We also performed a power spectrum for each patient. In addition, we similarly performed a spectral analysis on 4 of the over-mentioned narcoleptic patients recorded during lucid dreaming, for whom we extracted lucid REM sleep samples, following a left-right-left-right ocular signal, as already explained in our previous work, ${ }^{12}$ and selected equivalent fragments of quiet closed-eyes wakefulness and non-lucid REM sleep.

\section{RESULTS}

\section{Case-reports}

Patient 1: Two episodes of sleep paralysis framed by ocular signals

This 18-year-old man was referred for a 5-year history of hypnagogic sleep paralysis, sometimes accompanied by intruder hallucinations as well as visual, auditory or tactile hallucinations, a mild sleepiness (Epworth sleepiness score: 11/24) and no cataplexy or sleep attacks. He was eventually diagnosed with a borderline form of narcolepsy type 2 (mean sleep onset latency of 10.4 min at the MSLT-when normal values are greater than 8 min- 3 of them containing REM sleep). However, as his main problem was recurrent sleep paralyses, and as they may occur during daytime naps, we asked him to signal any sleep paralysis via the ocular code commonly used in lucid dreaming (i.e., left-rightleft-right large horizontal eye movements), as we previously did in patients with narcolepsy. ${ }^{12}$ After the three naps containing REM sleep, he reported having experienced a sleep paralysis, which had been signaled in two of the three naps by the 
ocular code decided before the nap. In particular, $30 \mathrm{sec}$ after entering REM sleep, he made a left-right-left-right ocular signal (Figure 2), marking the beginning of the sleep paralysis episode, which lasted 30 seconds before awakening. He had an analogous episode during the fourth nap. Seventy seconds after entering into REM sleep, he signaled the beginning of the paralysis, which lasted 113 seconds before awakening. Afterwards, the patient explained that he was not dreaming but had sleep paralysis.

\section{Patient 2: A sleep paralysis reported upon awakening}

A 48-year-old woman with a history of obstructive sleep apnea syndrome adequately treated with continuous positive airway pressure was referred for residual excessive daytime sleepiness (Epworth sleepiness score: 17/24). She also reported rare hypnagogic hallucinations and episodes of sleep paralysis with an intruder hallucination (feeling the presence of unknown people in her room). She was eventually diagnosed with a borderline form of narcolepsy type 2 (no cataplexy, mean sleep latency at the MSLT: 8.9 minutes; 4 SOREMPs /5 naps). At the end of the second nap, she reported to the nurse having experienced a sleep paralysis just before awakening.

Patient 3: Isolated sleep paralysis followed by a false awakening, time-marked by the National Day's fireworks

A 27-year-old woman presented a 5 year-long history of hypnagogic (and rarely hypnopompic) hallucination, accompanied by sleep paralysis, in absence of other sleep complaints. During the sleep paralysis episodes, she reported seeing a black demon moving and speaking with an incomprehensible language, feeling a thoracic oppression and sometimes feeling a presence in her room (like a thief). She met the diagnosis of recurrent isolated sleep paralysis. During the first night of sleep monitoring, at the end of the first cycle of REM sleep, we recorded a sleep paralysis lasting 5 minutes (300 
seconds) before awakening (Figure 3). The exact time of sleep paralysis was accurate, because the episode started (as later reported) at time of the first detonation of the fireworks of the National Day on the Eiffel tower of Paris, France (the exact time of fireworks is scheduled and can also be heard on the audio of the polysomnography). At the video inspection during sleep paralysis, she was lying in dorsal position, had initial lips movements without sound emission, followed by moaning at different times during the episode, as if she was trying to call someone with the mouth closed. In the middle of the episode, she briefly opened her eyes. Finally, she managed to perform some small jerky movements of the feet, followed by the arms and then woke up. After the episode, she recalled having experienced a burning sensation widespread over the body, having tried to emit vocal sounds because of the impossibility to move. She also mentioned to have experienced a false (dreamed) awakening at the end of her sleep paralysis episode, (falsely) recalling that she succeeded to push the button for calling the nurse, who finally came and touched her hand, achieving to wake her up.

\section{Patient 4. Feeling crushed by the physician}

An 18-year-old woman was referred for excessive daytime sleepiness (Epworth sleepiness score: 16/24) and sleep attacks. She sometimes experienced hypnopompic hallucinations with feelings of presence of people or shadows. She had a normal (12.5 minutes) sleep latency at the MSLT but 3 SOREMPs, and a SOREMP during the "ad libitum" day of recording (patients are monitored during a first night, followed by MSLT and followed again by ad libitum night and day sleep monitoring, (see the bed rest procedure in ${ }^{14}$ ). When awakening from the daytime nap's SOREMP, she reported having experienced a sleep paralysis associated with the hallucination of a physician crushing her and trying to suffocate her. 


\section{Patient 5. False awakening as an isolated sleep state misperception}

A 22-year-old woman complained of excessive daytime sleepiness (Epworth sleepiness score: 18/24) and irresistible sleep attacks at school or when driving a car. She suffered from cataplexies, disturbed night sleep and frequent nightmares, lucid dreaming, false awakenings, frequent sleep paralyses as well as visual, auditory and presence hypnagogic hallucinations (including out of body experiences). She accurately distinguished these various dissociate sleep-wake states. As an example of false awakening, she reported: "I wake up and leave my bed. I eat my breakfast, wash my hair, I prepare my bag. I leave my flat, go down in the street, take my bus. And then in the bus, I realize that something is wrong, something about the time is wrong. Still in my bus, I wake up and realize this was only a dream. In other instances, I do all this morning routine until taking my bus and arriving at school, and then at school I realize that I am alone, the school is empty. This looks so weird that I wake up and realize this was a dream". Sleep paralysis are described as hypnagogic episodes, often associated with a weird tinnitus: "I try to fall asleep when hearing this tinnitus, turn to be supine in the bed, and then I think that I open my eyes, and despite it is dark around in the room, I am still able to see a person arriving in front of me. I start to be very afraid, try to move, shout and call for help. I feel that I cannot move of shout. Then I try to calm myself, by I see a passing shadow that comes from the ceiling or the door (this is generally a shadow, but sometimes it can be Lord Voldemort from the Harry Potter book himself, or just a flying face with a wide-open mouth coming on me). The shadow stays close to me or in the wood of my bed, or is on me and tries to catch me". She met the criteria of narcolepsy type 1 (MSLT latency: 7.9 minutes; 4 SOREMPs). At the beginning of the first nap, she reported a false awakening. She dreamed of lying in the bed at the sleep laboratory, during the sleep monitoring, trying to fall asleep and saying to herself: "You have to sleep, you have to sleep". She realized when she woke up that it was a dream (a 
"false waking") and that she was already sleeping. During this nap (sleep onset latency of $6.5 \mathrm{~min}$ ), she had a late and brief SOREMP, entering from wakefulness directly into REM sleep for a duration of 1 minute, before awakening and realizing she had experienced a false awakening.

\section{Visual polysomnographic aspects of the episodes}

An accurate timing of the episodes is stateable for Patient \#1 (ocular code), Patient \#3 (video-recording with auditory trigger) and Patient \#5 (direct entering in REM sleep for 60 seconds before awakening). For Patients \#2 and \#4, because of the impossibility to document the exact beginning of the episodes, we inspected the 60 seconds before awakening, being aware of the arbitrariness of this choice.

All patients had the episodes during REM sleep and ended with awakening, when they reported the experience to the nurses. The episodes occurred during daytime naps in Patients \#1, \#2, \#4 and \#5, and during night-time in Patient \#3. All episodes occurred during a SOREMP, with the exception of Patient \#3. The visual inspection of the EEG background for all patients showed a theta predominance, with some bursts of alpha rhythm; in particular, these were in correspondence with REMs in Patient \#1 and with the attempts of vocalizations in Patient \#3. The EOG showed the presence in all patients of isolated REMs, sometimes occurring in brief bursts. The only exception was Patient \#1, which performed the voluntary left-right-left-right signal, marking the beginning of the sleep paralysis, followed by bursts of usual REMs before the definite awakening (Figure 2). The EMG recorded on the chin muscle displayed a complete muscle atonia in all subjects, with rare and subtle activations in some cases (in coincidence with the ocular code in Patient \#1, and during the last seconds of the episode in Patient \#3, when she began to move before definitely awakening. 


\section{The 3 seconds mini-epochs analysis during the sleep paralysis}

The results of the 3-second mini-epochs (over a total of 680 mini-epochs) analysis are shown in Table 1. As regards to EEG visual inspection of the 3-second mini-epochs, the relative percentage of the alpha band during sleep paralysis was lower than during wakefulness and higher than during normal REM sleep $(\mathrm{P}<0.001)$, setting the sleep paralysis condition in an intermediate position between the two states. This intermediate position was also found when comparing the spectral analysis between the three conditions (Figure 1). Compared to lucid REM sleep, there were increased amounts of EEG alpha rhythm during sleep paralysis $(\mathrm{P}=0.001)$. Concerning the chin muscle tone, beyond the expected differences regarding enhanced muscle tone between sleep paralysis $v s$. wakefulness and REM sleep $v s$. wakefulness $(\mathrm{P}<0.001)$, no further difference was found between sleep paralysis and normal REM sleep. However, there were more frequent mini-epochs with enhanced muscle tone during lucid REM sleep than during sleep paralysis $(\mathrm{P}=0.019)$. Regarding the number and type of ocular movements, no difference was identified between the different states.

\section{Spectral EEG analysis of sleep paralyses, false awakenings and lucid REM sleep}

Regarding spectral analysis (Figure 1), the patients experienced sleep paralyses during fragments of different durations (113 seconds in Patient \#1, 300 seconds in Patient \#3 and 60 seconds in Patient \#4). We could not analyse the measures in Patient \#2 because of an artefact on the EEG channels. As illustrated in Figure 1A, the global power spectrum of the experience of sleep paralysis (using a group analysis in these 3 patients) showed an intermediate power spectrum between wakefulness and REM sleep in the low frequencies and in the alpha band, whereas the more rapid beta EEG frequencies were not different between sleep paralysis and normal REM sleep. 
The spectral analysis of false awakenings (Figure 1B) was performed during the last 30 seconds before awakening in Patient \#3 (even if we are aware of the arbitrariness of this choice), and during the 60 seconds of the isolated false waking in Patient \#5. The spectral power appears in all respects similar to that of sleep paralysis, with the same intermediate position (between wakefulness and normal REM sleep) of low frequencies and alpha band and a profile of the beta band similar to that of normal REM sleep. Eventually, the spectral analysis of lucid REM sleep was not different from that of nonlucid REM sleep (Figure 1C).

\section{DISCUSSION}

The current classification of sleep disorders places sleep paralysis as a dissociated state between normal REM sleep and wakefulness, mostly considering muscle atonia (a characteristic of REM sleep), in contrast with an "awake" subject, which is conscious of the surrounding environment. However, it is quite common during the episode to experience concomitant multi-sensorial hallucinations, which are considered as fragments of dream contents intruding into wakefulness. ${ }^{12}$ This widely diffused concept of a preserved alertness in an atonic body ${ }^{15}$ during sleep paralysis can however now be challenged in many aspects.

\section{The brain is not awake during sleep paralysis}

First of all, at visual inspection, in contrast with some previous reports describing an almost awake EEG tracing with bursts of REMs and muscle atonia, ${ }^{4}$ the five recorded sleep paralyses here were associated with EEG features typical of REM sleep (not of wakefulness), including predominant theta rhythms, and occasional bursts of alpha rhythms (concomitant with rapid eye movements) as well as muscle atonia. Consequently, the concept of the sleep paralysis as an awake brain in a paralyzed body 
might be reviewed in favour of a rather partially aware but dreaming state, as already postulated in some studies. ${ }^{1}$ Both the spectra and the 3 -seconds analysis of the recorded paralysis episodes here display comparable features with the previous case-report by Terzaghi et al., ${ }^{6}$ setting EEG during sleep paralysis in an intermediate state between REM sleep and wakefulness, with an intermediate low frequencies EEG spectrum, an intermediate power in the alpha band and a profile more similar to that of normal REM sleep regarding higher (beta) frequencies. Our results confirm their initial findings in a higher number of sleep paralysis episodes, some of them being more accurately signalled than in previous works, thanks to an external (the firework) or an internal (the eye code) markers.

\section{'Lucid' sleep paralysis}

To our knowledge, this is the first time that the ocular coding (currently used for signalling lucid dreaming in REM sleep), is obtained for signalling an episode of sleep paralysis, thus configuring for the first time a "lucid" sleep paralysis. Of interest, if the muscle atonia spares the voluntary oculomotor muscles during REM sleep, it seems also to spare them during sleep paralysis, confirming objectively some reports of patients being able to move their eyes (and sometimes to open them) during sleep paralysis. This patient, even being "conscious" of his state of paralysis showed a typical REM sleep EEG, similarly to lucid dreaming (when asleep people are aware of dreaming). Notwithstanding, his EEG power spectrum showed a peak in the alpha band between wakefulness and REM sleep, similarly to the other sleep paralysis episodes, in sharp contrast with lucid REM sleep in lucid dreamers (in whom the spectra appear utterly similar to proper REM sleep here). The experience of lucid dreaming, in fact, seems to display a reduction in delta frequencies in comparison with non-lucid REM sleep, ${ }^{12}$ but no difference in the alpha band, characteristics which appears peculiar to both 
experiences of sleep paralysis and, with proper limitations, false awakening. Of interest, lucid dreamers do not feel paralyzed during lucid REM sleep (despite their body is really paralyzed), whereas patients with sleep paralysis feel the real body paralysis (despite their brain is partly in a REM sleep state). The inputs from the muscle sensors to the brain sensory cortex seem to be ungated during sleep paralysis but blocked during lucid dreaming, suggesting local variations in cortex connectivity among dissociated states.

\section{False awakenings}

Clinical and neurophysiological descriptions of false awakenings are scarce. The single report by Takeuchi et al., ${ }^{11}$ later considered as a false awakening by other authors, ${ }^{9}$ depicts an hypnagogic hallucination of unpleasant and fearful feeling of presence in the sleep laboratory with the perception of having rose from the bed. The polysomnography showed abundant trains of alpha rhythm on the EEG, sometimes blocked by REMs mixed with slow eye movements and low muscle tone. Conversely, the two experiences of false awakening monitored here (similarly to sleep paralysis) were close to regular REM sleep. Indeed, the visual EEG inspection and quantitative analysis clearly shows predominant theta rhythms, suggesting that these two experiences are the products of a dreaming rather than a fully conscious brain. This confirms a strong link between the two experiences, which may sometimes continue into one another, as in Patient \#3, and as underlined in a previous population survey. ${ }^{9}$ However, given the impossibility to accurately time-mark the beginning of the false awakening in Patient \#3, these results need to be reproduced.

\section{What do these results tell us about sleep paralysis?}


The intermediate features between REM sleep and wakefulness of both sleep paralysis and false awakening might be explained in light of the currently consolidated theories of local sleep, stating that different sleep phases or wakefulness, especially in the transitional states, can show intermingled features with one another. ${ }^{16}$ In this direction, the night-time sleep of subjects with recurrent idiopathic sleep paralysis contains higher delta power than healthy subjects, suggesting an alteration in wakefulness-NREM-REM sleep regulation in sleep paralysis sufferers. ${ }^{1}$ Notably, all but Patient \#3 experienced the episodes during SOREMPs, a timing where sleep mechanisms of phase transition may be altered, possibly favouring local REM sleep phenomena In fact, previous studies have ascertained that SOREMPs facilitate the onset of sleep paralysis, in patients with narcolepsy as well as in healthy subjects. ${ }^{4,5}$ This aspect may also explain why patients with narcolepsy experience sleep paralysis more frequently than healthy subjects do. Regarding false awakening, the single previous description was recorded during a SOREMP,${ }^{11}$ as here in Patient \#5, whereas in Patient \#3 the false awakening emerged from a sleep paralysis. Sleep paralysis and false awakenings share the feeling of being in a familiar environment (usually the bed/sofa in one's house) and not in another place as when dreaming, the perception of being awake, and frequent feelings of anxiety, ${ }^{1}$ but inability to move during sleep paralysis episodes $v s$. freedom to move during false awakening episodes (Table 3). In this respect, sleep paralysis, which is often associated with supine body position, has been linked to disturbances in the parietal cortex, where monitoring of motor programs through proprioceptive feedback normally takes place. During sleep paralysis, the persistent muscle atonia can create a mismatch contributing to abnormal physical perceptions and modifying the self-body image. ${ }^{1,17}$

\section{Limitations}


Because only three EEG channels were used for routine sleep recordings, we cannot topographically localize the power spectra source over the EEG scalp. It will be highly desirable in future studies to capture these experiences with a more extensive EEG montage, in order to better elucidate the spatial scalp EEG correlates of these fascinating phenomena. Another limitation here is the reduced sample, which is expected knowing the difficulty of capturing these rare experiences. Eventually, two of the subjects with false awakenings and sleep paralyses (and the four lucid dreamers) had a narcolepsy, which may restrain the generalization of the findings to the healthy population. Moreover, we are aware that the arbitrary choice of inspecting the 60 seconds before the awakening in two patients, may represent a further limitation. Eventually, we could not capture the three phenomena (sleep paralysis, false awakening and lucid REM sleep) in the same subjects, preventing more precise within-subjects' comparisons of the three experiences.

Although our results may be speculative in some aspects, their principal strength is that episodes of sleep paralysis in different subjects show a similar visual aspect and spectral profile on EEG, in accordance with a previous single report using spectral analysis. ${ }^{6}$ Besides, we provided the first report of a 'lucid' sleep paralysis (a robust, time marked episode) and a spectral analysis of two false awakenings, even if these last results are less reliable.

\section{References}

1. Fox K, Christoff K. The Oxford Handbook of Spontaneous Thought: Mind-Wandering, Creativity, and Dreaming. Oxford, UK: Oxford University Press, 2018.

2. American Academy of Sleep Medicine. The international Classification of Sleep Disorders, 3rd edition. Darien, IL: American Academy of Sleep Medicine, 2014. 
3. Denis D, French CC, Gregory AM. A systematic review of variables associated with sleep paralysis. . Sleep Med Rev 2018;38:141-57.

4. Takeuchi T, Miyasita A, Sasaki Y, Inugami M, Fukuda K. Isolated sleep paralysis elicited by sleep interruption. Sleep 1992:217-25.

5. Takeuchi T, Fukuda K, Sasaki Y, Inugami M, Murphy TI. Factors related to the occurrence of isolated sleep paralysis elicited during a multi-phasic sleep-wake schedule. Sleep 2002;25:89-96.

6. Terzaghi M, Ratti PL, Manni F, Manni R. Sleep paralysis in narcolepsy: more than just a motor dissociative phenomenon? Neurol Sci 2012;33:169-72.

7. Pizza F, Moghadam KK, Franceschini C, Bisulli A, Poli F, Ricotta L, et al. Rhythmic movements and sleep paralysis in narcolepsy with cataplexy: a video-polygraphic study. Sleep Med 2010;11:423-5.

8. Stefani A, Holzknecht E, Hogl B. Clinical neurophysiology of REM parasomnias. Handb Clin Neurol 2019;161:381-96.

9. Buzzi G. False awakenings in light of the dream protoconsciousness theory: a study in lucid dreamers. Int J Dream Res 2011;4:110-6.

10. Nielsen T, Zadra A. (2011): Idiopathic nightmares and dream disturbances associated with sleep-wake transitions. In: Kryger M, Roth T, Dement W, eds. Principles and Practice of Sleep Medicine. Vth ed: Elsevier:1106-15.

11. Takeuchi T, Miyasita A, Inugam M, Sasaki Y, Fukuda K. Laboratory-documented hallucination during sleep-onset REM period in a normal subject. Percept Mot Skills 1994;78:979-85.

12. Dodet P, Chavez M, Leu-Semenescu S, Golmard J, Arnulf I. Lucid dreaming in narcolepsy. Sleep 2015;38:487-97.

13. Oudiette D, Dodet P, Ledard N, Artru E, Rachidi I, Similowski T, et al. REM sleep respiratory behaviours match mental content in narcoleptic lucid dreamers. Sci Rep 2018;8:2636-46. 
14. Vernet C, Arnulf I. Idiopathic hypersomnia with and without long sleep time: a controlled series of 75 patients. Sleep 2009;32:752-9.

15. Hishikawa Y, Shimizu T. Physiology of REM sleep, cataplexy, and sleep paralysis. Adv Neurol 1995;67:245-71.

16. Mahowald MW, Schenck CH. Insights from studying human sleep disorders. Nature $2005 ; 437: 1279-85$.

17. Jalal B. How to make the ghosts in my bedroom disappear? Focused-attention meditation combined with muscle relaxation (MR Therapy) — A direct treatment intervention for sleep paralysis. Front Psychol 2016;7:28. 


\section{Legend of the Figures}

Figure 1 - Global EEG power spectrum: a) during five episodes of sleep paralysis (SP, black line) vs. wakefulness (red line) and REM sleep (blue line) in three patients; b) during two episodes of false awakening (FA, green line) vs. REM sleep (blue line) and wakefulness (red line) in two patients ; c) during lucid REM sleep (orange line), vs. REM sleep (blue line) and wakefulness (red line) in 5 other, different patients with narcolepsy.
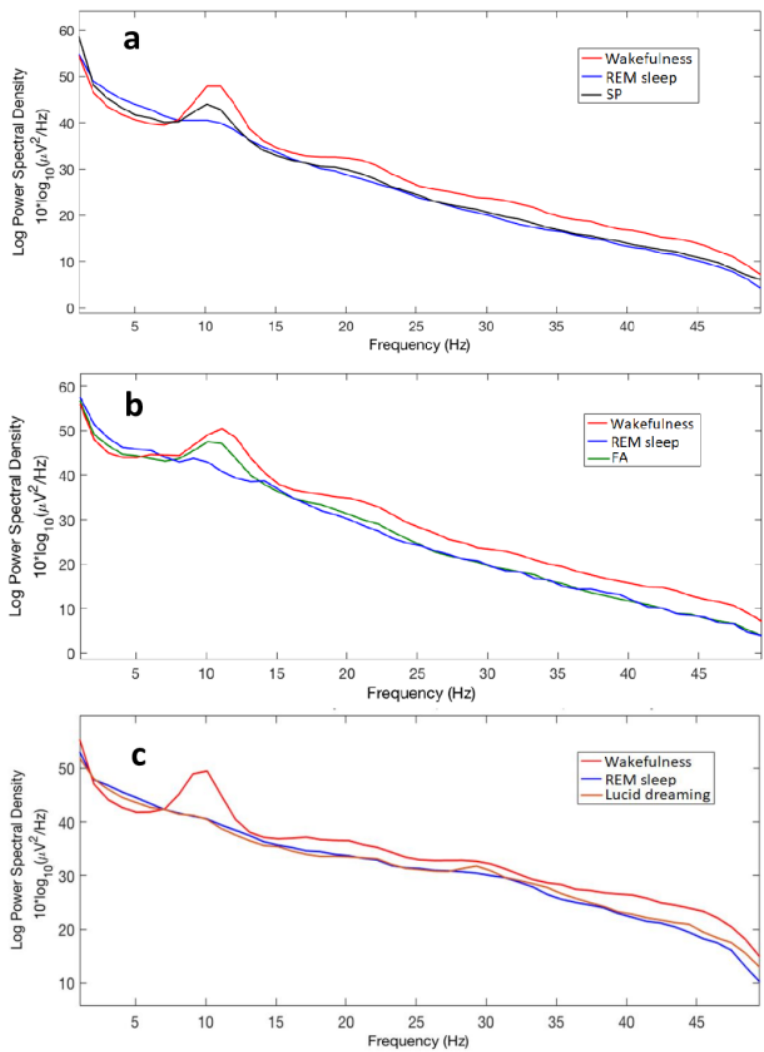
Figure 2 - An episode of sleep paralysis signaled by the left-right-left right (LRLR ocular coding, vertical arrow) horizontal eye movements (the same code is often used to signal lucid dreaming in lucid dreamers), with clear theta rhythms on the EEG, as shown here in a $30 \mathrm{sec}$ epoch of polysomnography. From top to bottom, EEG (Fp1/A2, C3/A2 and C3/O1 channels with their filters and amplitudes), electro-oculography (EOG 1: right superior canthus/A2; EOG2: left inferior canthus/A2) and electromyography (EMG1). Green vertical lines rule every second of clock time.

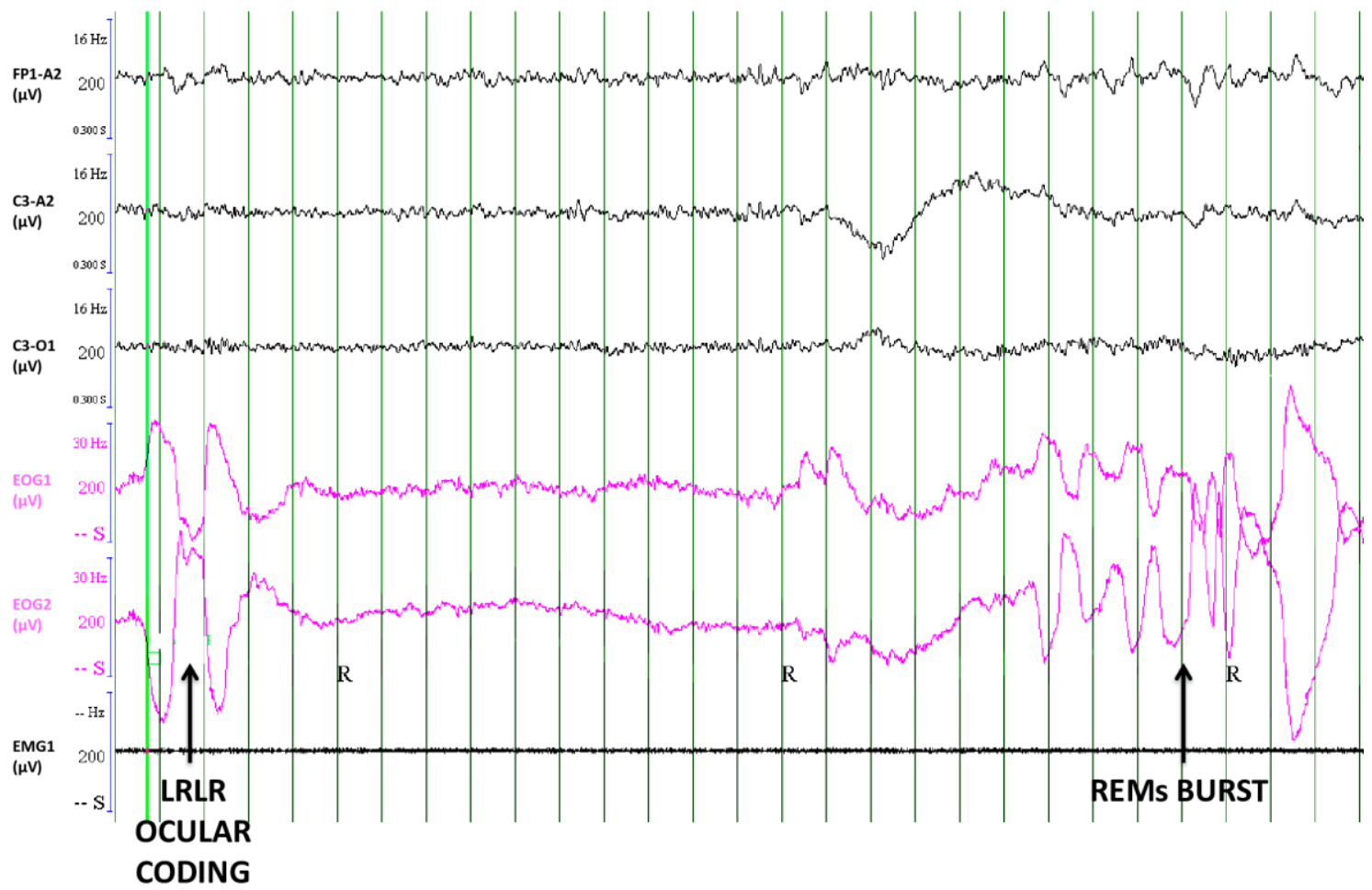

Figure 3 - Polysomnography monitoring during a 5 min long sleep paralysis. The upper part of the figure displays the neurophysiological channels of a single $30 \mathrm{sec}$ long epoch, corresponding below to the period shown in black brackets (from top to bottom; EEG channels in black color, Fp1/A2, C3/A2 and O1/; electro-oculography EOG right and left in red color, and EMG of the chin (green) and right and left tibialis anterior (purple); the lower part correspond to 5 min of the respiratory and heart monitoring 
during the full episode of sleep paralysis, with corresponding visible events in black boxes.

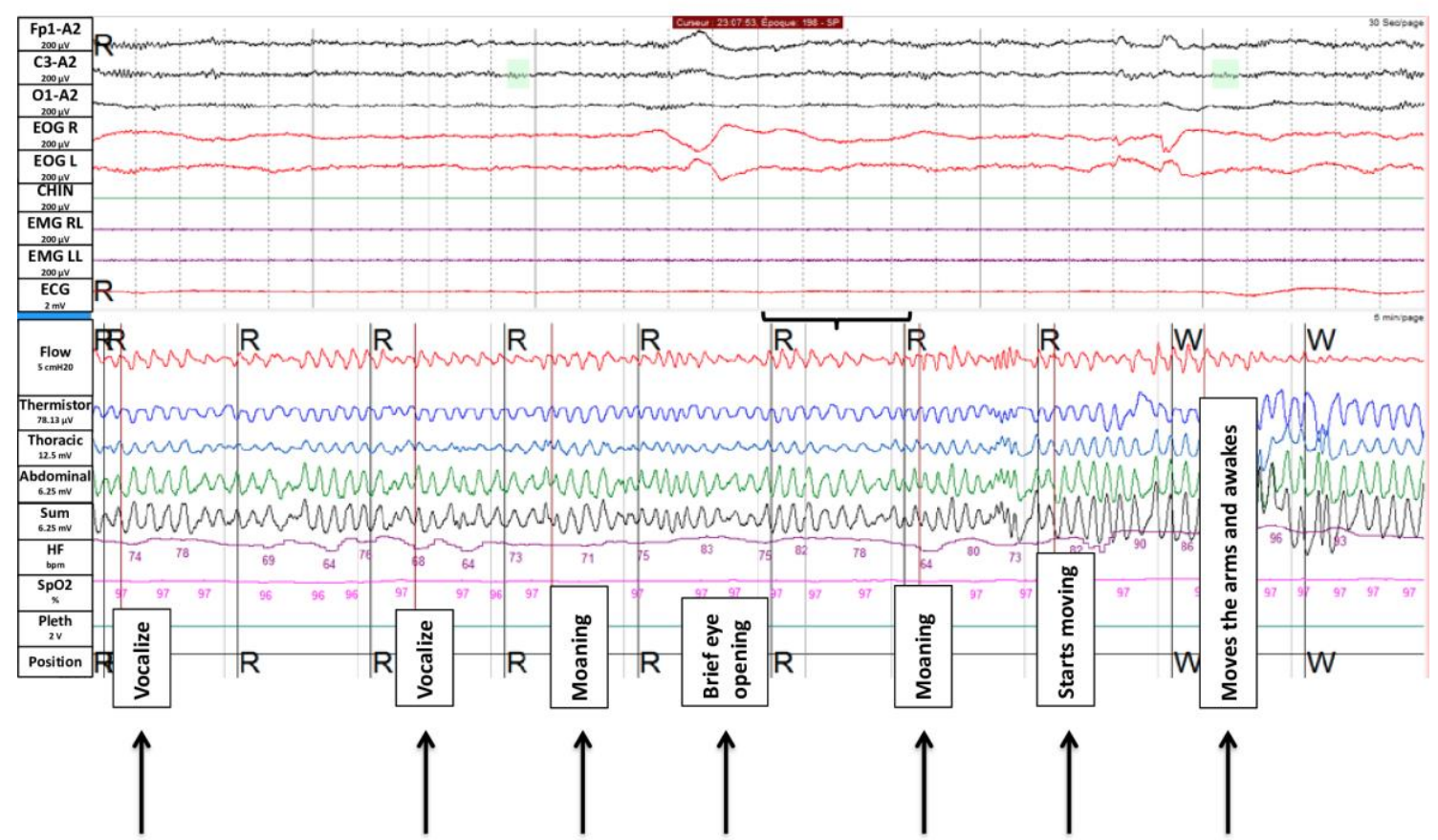


Table 1 - Quantification of EEG, EMG and EOG characteristics via analyzing 3 sec-mini-epochs during REM sleep, wakefulness, sleep paralysis and lucid dreaming episodes

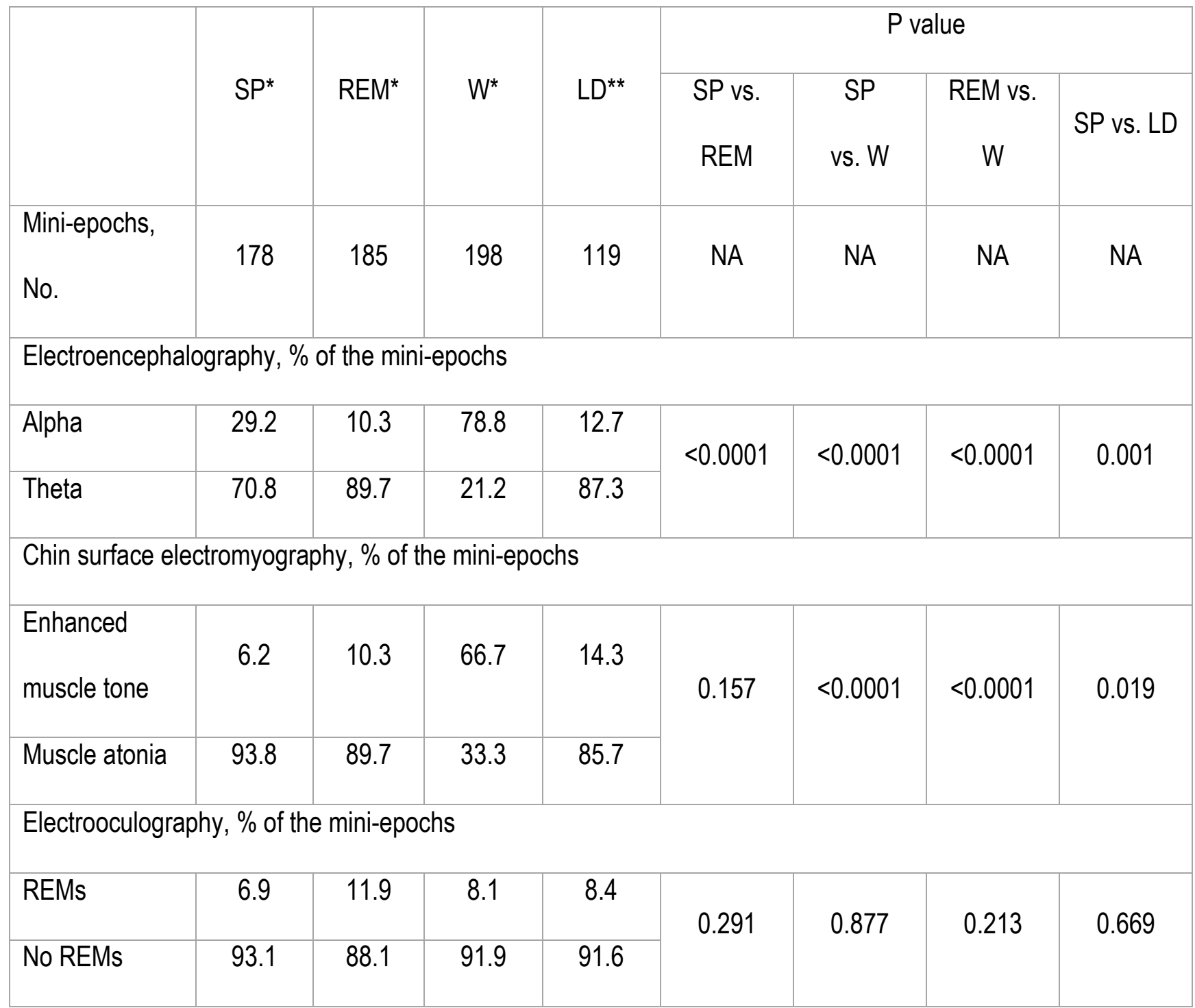

* These 3 sec mini-epochs are extracted from the measures in Patient 1 and in Patient 3; **

These 3-seconds mini-epochs of lucid REM sleep are extracted from 4 patients with narcolepsy.

LD: lucid dreaming; REM: REM sleep; REMs: rapid eye movements, SP: sleep paralysis; W:

wakefulness 
Table 2 - Clinical and neurophysiological characteristics of sleep paralysis, false awakenings and lucid dreaming, compared to normal wakefulness and REM sleep

\begin{tabular}{|c|c|c|c|c|c|}
\hline & $\begin{array}{c}\text { Wakefulness, } \\
\text { closed eyes }\end{array}$ & $\begin{array}{c}\text { Sleep } \\
\text { paralysis }\end{array}$ & $\begin{array}{c}\text { False } \\
\text { awakening }\end{array}$ & $\begin{array}{l}\text { Lucid } \\
\text { dreaming }\end{array}$ & REM sleep \\
\hline \multicolumn{6}{|l|}{ Clinical features } \\
\hline Feeling paralyzed & No & Yes & No & No & No \\
\hline $\begin{array}{l}\text { Feeling the } \\
\text { surroundings as } \\
\text { familiar }\end{array}$ & Yes & Yes & Yes & No & No \\
\hline $\begin{array}{l}\text { Accurate awareness } \\
\text { of the state }\end{array}$ & Yes & Yes & No & Yes & No \\
\hline Associated anxiety & None & Frequent & Frequent & None & None \\
\hline Mentation & $\begin{array}{l}\text { Awake } \\
\text { thoughts }\end{array}$ & $\begin{array}{c}\text { Feels awake } \\
\text { but may feel } \\
\text { hallucinations }\end{array}$ & Feels awake & Dreaming & Dreaming \\
\hline \multicolumn{6}{|l|}{ EEG } \\
\hline Delta band & Low & $\begin{array}{l}\text { Similar to } \\
\text { REM sleep }\end{array}$ & $\begin{array}{l}\text { Similar to } \\
\text { REM sleep }\end{array}$ & $\begin{array}{l}\text { Lower than } \\
\text { REM sleep }\end{array}$ & High \\
\hline Theta band & Low & $\begin{array}{l}\text { Similar to } \\
\text { REM sleep }\end{array}$ & $\begin{array}{l}\text { Similar to } \\
\text { REM sleep }\end{array}$ & $\begin{array}{l}\text { Similar to } \\
\text { REM sleep }\end{array}$ & High \\
\hline Alpha band & High & $\begin{array}{l}\text { Intermediate } \\
\text { between Rem } \\
\text { sleep and } \\
\text { wakefulness }\end{array}$ & $\begin{array}{c}\text { Intermediate } \\
\text { between } \\
\text { Rem sleep } \\
\text { and } \\
\text { wakefulness }\end{array}$ & $\begin{array}{l}\text { Similar to } \\
\text { REM sleep }\end{array}$ & Low \\
\hline Beta-Gamma bands & High & $\begin{array}{l}\text { Similar to } \\
\text { REM sleep }\end{array}$ & $\begin{array}{l}\text { Similar to } \\
\text { REM sleep }\end{array}$ & $\begin{array}{c}\text { Similar to } \\
\text { wakefulness } \\
\text { (frontal lobe) } \\
\text { in one study } \\
\text { and similar to } \\
\text { REM sleep in } \\
\text { another study }\end{array}$ & Low \\
\hline
\end{tabular}




\begin{tabular}{|l|c|c|c|c|c|}
\hline EMG & High & $\begin{array}{c}\text { Similar to } \\
\text { REM sleep }\end{array}$ & $\begin{array}{c}\text { Similar to } \\
\text { REM sleep }\end{array}$ & $\begin{array}{c}\text { Similar to } \\
\text { REM sleep }\end{array}$ & Low \\
\hline EOG & Present & Present & Present & Present & Present \\
\hline Spontaneous REMs & Present & Present & Possible & Present & Absent \\
\hline $\begin{array}{l}\text { Voluntary REMs } \\
\text { (eye code) }\end{array}$ & & & & \\
\hline
\end{tabular}

